\title{
CONJUGATION SPACES AND 4-MANIFOLDS
}

\author{
IAN HAMBLETON AND JEAN-CLAUDE HAUSMANN
}

\begin{abstract}
We show that 4-dimensional conjugation manifolds are all obtained from branched 2-fold coverings of knotted surfaces in $\mathbb{Z}_{2}$-homology 4 -spheres.
\end{abstract}

\section{INTRODUCTION}

Flag manifolds $X$ with complex conjugation, the Chevalley involution on coadjoint orbits of compact Lie groups, and involutions on toric manifolds or polygon spaces all share a remarkable property. Let $G$ denote the group of order 2 . There is a ring isomorphism

$$
\kappa: H^{2 *}\left(X ; \mathbb{Z}_{2}\right) \cong H^{*}\left(X^{G} ; \mathbb{Z}_{2}\right)
$$

dividing the degrees in half, where $X^{G}$ denotes the fixed set under the involution. The structure underlying this property was discovered by Hausmann, Holm and Puppe [23], and studied further in [16, 22, 35, 36, 37. A $G$-space with this structure is a conjugation space (see Section 2 for the precise definition).

In this paper, we study the interaction between conjugation structures and the topology of smooth 4-manifolds. A conjugation 4-manifold is a smooth closed $G$-manifold $X$ of dimension 4 which is a conjugation space. The fixed point set $X^{G}$ is a closed connected surface embedded in $X$. In addition, $X$ has no odd-degree cohomology $(\bmod 2)$, and hence a conjugation manifold is orientable.

Let $X$ be an oriented conjugation 4-manifold. The quotient space $X / G$ inherits a canonical smooth structure (see Lemma 7.5), and thus $X / G$ is an oriented closed smooth 4-manifold containing the surface $X^{G}$ as a smooth submanifold.

A $\mathbb{Z}_{2}$-knot is a smooth manifold pair $(M, \Sigma)$, where $M$ is an oriented 4-dimensional $\mathbb{Z}_{2}$-homology sphere and $\Sigma$ is a closed connected surface embedded in $M$.

Theorem A. The correspondence $X \mapsto\left(X / G, X^{G}\right)$ defines a bijection between

(a) the orientation-preserving G-diffeomorphism classes of oriented connected conjugation 4-manifolds, and

(b) the smooth equivalence classes of $\mathbb{Z}_{2}$-knots.

Two $\mathbb{Z}_{2}$-knots $(M, \Sigma)$ and $\left(M^{\prime}, \Sigma^{\prime}\right)$ are smoothly equivalent if there is an orientationpreserving diffeomorphism $h: M \rightarrow M^{\prime}$ such that $h(\Sigma)=\Sigma^{\prime}$.

The inverse of the bijection in Theorem A is provided by taking a branched 2-fold covering of $M$ over the knot $\Sigma$. We therefore need to understand the relation between

Date: June 8, 2010.

Research partially supported by NSERC Discovery Grant A4000. The first author would like to thank the Max Planck Institut für Mathematik in Bonn. Both authors thank the Swiss National Funds for Scientific Research for its support. 
smooth manifold structures on the total spaces and quotients of branched 2-fold coverings, with codimension two branch locus (see the Appendix 877). Other versions of Theorem A are given in Section 6, for instance for topological manifolds (Theorem B), or for nonoriented manifolds.

Under the bijection of Theorem A, any knot $S^{2} \hookrightarrow S^{4}$ corresponds to a conjugation 4-manifold $X$ with $X^{G} \approx S^{2}$. For the trivial knot $S^{2} \subset S^{4}, X$ is the sphere $S^{4}$ on which $G$ acts by a linear involution with 2 negative eigenvalues (see Example 4.1). In general, $X$ is not simply connected. On the other hand, Gordon [20], 21] and Sumners [44] found infinitely many topologically distinct knots in $S^{4}$ which are the fixed point set of smooth involutions (contrasting with the Smith conjecture in dimension 3), and earlier examples on homotopy 4-spheres were found by Giffen [19]. Our work adds a new perspective: the examples of Gordon and Sumners produce infinitely many topologically inequivalent smooth conjugations on $S^{4}$ (see Section 5 ).

The classical examples of conjugation 4-manifolds come from the complex conjugations on $S^{2} \times S^{2}$, with fixed point set $S^{1} \times S^{1}$, and on $\mathbb{C} P^{2}$ (or $\overline{\mathbb{C P}}^{2}$ ), with fixed point set $\mathbb{R} P^{2}$. By taking connected sums along the fixed sets, one can thus realize any closed surface as the fixed point set of a conjugation 4-manifold. These classical examples all have quotient a smooth manifold diffeomorphic to $S^{4}$ (see Arnold [3], Kuiper [27], Letizia [30], and Massey [33]). For the reader's convenience, in Proposition 5.4 we include a proof using classification results for group actions by Bredon [5] and Orlik-Raymond [39] (but not the deep results of Freedman [17] or Cerf [6]).

If $X$ is any simply-connected conjugation 4-manifold, we prove in Proposition 5.3 that $X / G$ is at least homeomorphic to $S^{4}$. In addition, we show in Proposition 2.19 that $X$ is homeomorphic to a connected sum of copies of $S^{2} \times S^{2}, \mathbb{C} P^{2}$, and $\overline{\mathbb{C P}}^{2}$ (but not necessarily equivariantly). For example, the K3 surface does not admit a conjugation structure.

Remark. A conjugation 4-manifold $X$ is equivariantly minimal among G-actions on 4manifolds with a given surface as the fixed set, since $X$ can not be decomposed as a non-trivial equivariant connected sum in the free part of the $G$-action (see Proposition 2.16).

For the remainder of the paper, the cohomology $H^{*}(-)=H^{*}\left(-; \mathbb{Z}_{2}\right)$ is taken with coefficients in the field $\mathbb{Z}_{2}$, unless otherwise mentioned. The letter $G$ stands for the group of order 2, with $G=\{1, \tau\}$, and a $G$-space is a space together with an involution $\tau$.

Acknowledgements. The authors would like to thank Allan Edmonds, Ron Fintushel, Cameron Gordon, Slava Kharlamov, Volker Puppe, Ron Stern and Claude Weber for helpful conversations and correspondence, and the referee for valuable suggestions.

\section{Conjugation spaces And MAnifolds}

For a $G$-space $X$, the equivariant cohomology $H_{G}^{*}(X)$ is defined as the (singular) cohomology of the Borel construction:

$$
H_{G}^{*}(X)=H^{*}\left(X \times_{G} E G\right) .
$$


Hence, $H_{G}^{*}(X)$ is a $H^{*}(B G)$-algebra via the projection $X \times_{G} E G \rightarrow B G$. Since $G$ is the group of order two, $B G=\mathbb{R} P^{\infty}$ and $H^{*}(B G)=\mathbb{Z}_{2}[u]$, with $u$ in degree 1 . Thus $H_{G}^{*}(X)$ is a $\mathbb{Z}_{2}[u]$-algebra. Let $\rho: H_{G}^{*}(X) \rightarrow H^{*}(X)$ and $r: H_{G}^{*}(X) \rightarrow H_{G}^{*}\left(X^{G}\right)$ be the restriction homomorphisms. As $G$ acts trivially on $X^{G}$, one has $\left(X^{G}\right)_{G}=B G \times X^{G}$, whence a canonical ring isomorphism $H_{G}^{*}\left(X^{G}\right)=H^{*}\left(X^{G}\right)[u]$.

2.1. Conjugation spaces ([23]). A cohomology frame or $H^{*}$-frame for a $G$-space $X$ is a pair $(\kappa, \sigma)$, where

(a) $\kappa: H^{2 m}(X) \rightarrow H^{m}\left(X^{G}\right), m \geq 0$, is an additive isomorphism dividing the degrees in half, and

(b) $\sigma: H^{2 m}(X) \rightarrow H_{G}^{2 m}(X), m \geq 0$, is an additive section of $\rho$.

Moreover, $\kappa$ and $\sigma$ must satisfy the conjugation equation

$$
r \circ \sigma(a)=\kappa(a) u^{m}+\ell_{m}(u)
$$

for all $a \in H^{2 m}(X)$ and all $m \in \mathbb{N}$, where $\ell_{m}(u)$ denotes any polynomial in the variable $u$ of degree less than $m$. An involution admitting a $H^{*}$-frame is called a conjugation. A $G$-space $X$ such that $H^{\text {odd }}(X)=0$ and admitting an $H^{*}$-frame is called a conjugation space.

Here below are some important properties of conjugation spaces.

(a) If $(\kappa, \sigma)$ is $H^{*}$-frame, then $\kappa$ and $\sigma$ are ring homomorphisms [23, Theorem 3.3]. The ring homomorphism $\kappa$ also commutes with the Steenrod squares: $\kappa \circ \mathrm{Sq}^{2 i}=$ $\mathrm{Sq}^{i} \circ \kappa$, [16, Theorem 1.3].

(b) $H^{*}$-frames are natural for $\tau$-equivariant maps [23, Prop. 3.11]. In particular, if an involution admits an $H^{*}$-frame, it is unique [23, Cor. 3.12].

(c) For a conjugate-equivariant complex vector bundle $\eta$ ("real bundle" in the sense of Atiyah) over a conjugation space $X$, the isomorphism $\kappa$ sends the total Chern class of $\eta$ onto the total Stiefel-Whitney class of its fixed bundle.

2.3. Equivariantly formal spaces. A $G$-space $X$ is equivariantly formal (over $\mathbb{Z}_{2}$ ) if the restriction homomorphism $\rho: H_{G}^{*}(X) \rightarrow H^{*}(X)$ is surjective. For instance, a conjugation space is equivariantly formal. The following result is proved in [2, Prop. 1.3.14].

Proposition 2.4. Let $X$ be finite dimensional $G-C W$-complex with $\sum b_{i}(X)$ finite, where $b_{i}(-)=\operatorname{dim} H^{i}(-)$. The following statements are equivalent.

(1) $X$ is equivariantly formal.

(2) $\sum b_{i}(X)=\sum b_{i}\left(X^{G}\right)$.

(3) The restriction homomorphism $H_{G}^{*}(X) \rightarrow H_{G}^{*}\left(X^{G}\right)$ is injective.

Remark 2.5. A smooth $G$-manifold has the equivariant homotopy type of a finite $G$ CW complex 25]. The assumptions of Proposition 2.4 are also satisfied for $X$ a closed topological manifold with a locally smooth G-action. See Kwasik [28] for this statement and further references.

Here is a consequence of Proposition 2.4.

Proposition 2.6. Let $X$ be a finite $G$-CW-complex. Suppose that $H^{*}(X) \approx H^{*}\left(S^{2 n}\right)$ and $H^{*}\left(X^{G}\right) \approx H^{*}\left(S^{n}\right)$. Then $X$ is a conjugation space. 
Proof. By Proposition 2.4, $X$ is equivariantly formal and the restriction homomorphism $r: H_{G}^{*}(X) \rightarrow H_{G}^{*}\left(X^{G}\right)$ is injective. Let $a \in H^{2 n}(X)$ and $b \in H^{n}\left(X^{G}\right)$ be the generators. Let $\sigma: H^{2 n}(X) \rightarrow H_{G}^{2 n}(X)$ be a section of $\rho: H_{G}^{2 n}(X) \rightarrow H^{2 n}(X)$. Since $H_{G}^{2 n}\left(X^{G}\right)$ is generated by $b u^{n}$ and $u^{2 n}$, one has $r \circ \sigma=\lambda_{1} b u^{n}+\lambda_{2} u^{2 n}$. Setting $\sigma^{\prime}(a)=\sigma(a)+\lambda_{2} u^{2 n}$ produces a new section $\sigma^{\prime}$ with $r \circ \sigma^{\prime}(a)=b u^{n}$. Hence, $X$ is a conjugation space.

2.7. Conjugation manifolds. A conjugation manifold is a smooth closed manifold equipped with a smooth involution which is a conjugation. As $H^{\text {odd }}(X)=0, X$ must be orientable and of dimension $2 n$. The fixed point set $X^{G}$ is a closed smooth manifold of dimension $n$. Also, the involution of a conjugation manifold preserves connected components (see [23, Remark 3.1]), so one can restrict to connected manifolds.

There are natural questions in all dimensions:

(i) Given a closed connected smooth manifold $M^{n}$, does there exists a conjugation $2 n$-manifold $X$ with $X^{G}$ diffeomorphic to $M$ ?

(ii) Given a closed connected smooth $2 n$-manifold $X$, does $X$ admit a smooth conjugation structure?

(iii) How can one classify conjugation manifolds up to G-diffeomorphism?

Theorem A provides an answer for question (iii) in dimension 4. The remainder of this section contains some partial results on questions (i) and (ii).

Remark 2.8. The circle is the fixed point of a unique conjugation 2-manifold, namely $S^{2}$ with a reflection through the equator. The uniqueness comes from the classical result that a continuous involution on $S^{2}$ is topologically conjugate to a linear one, see Constantin and Kolev [7, Theorem 4.1]. For a smooth conjugation, the uniqueness follows from the smooth Schönflies theorem. The work of Olbermann [35, 36, 37] addresses questions (i)-(iii) for 6-manifolds.

We have already seen that any closed surface can be the fixed point set of a conjugation manifold. However, the answer to question (i) can be negative without further assumptions on $M^{n}$, for $n>2$. For example, W. Pitsch and J. Scherer observed that the Cayley projective plane is a closed 16-dimensional manifold [46, Theorem 7.21, p. 707], which can not be the fixed point set of any conjugation space. Indeed, a famous theorem of Adams and its proof $[1$, Theorem 1.1 .1$]$ shows that $\mathbb{Z}_{2}[x] /\left(x^{3}\right)$ is not the $\mathbb{Z}_{2}$-cohomology ring of a space if degree $(x)>8$.

If $X$ is a manifold, we denote by $v_{i}(X)$ and $w_{i}(X)$ in $H^{i}(X)$ its $\mathrm{Wu}$ and Stiefel-Whitney classes. Proposition 2.9 below and its corollaries were also noticed by Pitsch and Scherer.

Proposition 2.9. Let $X$ be a smooth conjugation manifold of dimension $2 n$, with $H^{*}$ frame $(\kappa, \sigma)$. Then $\kappa\left(v_{2 i}(X)\right)=v_{i}\left(X^{G}\right)$ and $\kappa\left(w_{2 i}(X)\right)=w_{i}\left(X^{G}\right)$.

Proof. The Wu class $v_{2 i}(X)$ is characterised by the equation

$$
v_{2 i}(X) \smile a=\operatorname{Sq}^{2 i}(a) \text { for all } a \in H^{2 n-2 i}(X) .
$$

The ring isomorphism $\kappa: H^{2 *}(x) \rightarrow H^{*}\left(X^{G}\right)$ satisfying $\kappa \circ \mathrm{Sq}^{2 i}=\mathrm{Sq}^{i} \circ \kappa$, [16, Theorem 1.3]. Applying $\kappa$ to (2.10) thus gives

$$
\kappa\left(v_{2 i}(X)\right) \smile \kappa(a)=\mathrm{Sq}^{i}(\kappa(a)) \text { for all } a \in H^{2 n-2 i}(X) .
$$


As $\kappa$ is bijective, (2.11) implies that

$$
\kappa\left(v_{2 i}(X)\right) \smile b=\mathrm{Sq}^{i}(b) \text { for all } b \in H^{n-i}\left(X^{G}\right),
$$

which implies that $\kappa\left(v_{2 i}(X)\right)=v_{i}\left(X^{G}\right)$. As $H^{\text {odd }}(X)=0$, the $\mathrm{Wu}$ formula says that

$$
w_{2 i}(X)=\sum_{k=1}^{i} \mathrm{Sq}^{2 i-2 k} v_{2 k}(X) \text {. }
$$

Applying $\kappa$ to (2.12) and using that $\kappa\left(v_{2 i}(X)\right)=v_{i}\left(X^{G}\right)$, we get

$$
\kappa\left(w_{2 i}(X)\right)=\sum_{k=1}^{i} \mathrm{Sq}^{i-k} \kappa\left(v_{2 k}(X)\right)=\sum_{k=1}^{i} \mathrm{Sq}^{i-k} v_{k}\left(X^{G}\right) .
$$

By the $\mathrm{Wu}$ formula for $X^{G}$, this implies that $\kappa\left(w_{2 i}(X)\right)=w_{i}\left(X^{G}\right)$.

The following corollary may be compared with [10, Theorem 3]. Note that, if a conjugation manifold $X$ is spin, it has a unique spin structure since $H^{1}(X)=0$.

Corollary 2.13. Let $X$ be conjugation manifold of dimension $2 n$. Then $X$ is spin if and only if $X^{G}$ is orientable.

Proof. As $H^{\text {odd }}(X)=0, X$ is spin if and only if $w_{2}(X)=0$. The results thus follows from Proposition 2.9.

Another corollary concerns non-oriented bordism.

Corollary 2.14. Let $X$ be a conjugation manifold. Then $X$ bounds a compact (possibly non-oriented) manifold if and only if $X^{G}$ does so.

Proof. By theorems of Pontrjagin and of Thom [34, pp. 52-53], a manifold bounds if and only if all its Stiefel-Whitney numbers vanish. As $H^{\text {odd }}(X)=0$, Proposition 2.9 implies that the collections of the Stiefel-Whitney numbers for $X$ and $X^{G}$ are in bijection.

Since a surface bounds if and only if its Euler characteristic is even, the same statement holds true, by Corollary 2.14, for a conjugation 4-manifold. Actually, any orientable 4-manifold satisfies $w_{2}^{2}=w_{4}$ by Wu's formula, so it bounds if and only if its Euler characteristic is even.

The following proposition will be useful.

Proposition 2.15. Let $X$ be a smooth closed connected G-manifold of dimension $4 n$. Suppose that $H^{k}(X)=0$ for $0<k<2 n$. Then, the following statements are equivalent

(1) $X$ is a conjugation manifold.

(2) $X^{G}$ is a 2n-manifold and $b_{n}\left(X^{G}\right) \geq b_{2 n}(X)$.

Observe that, in general, the existence of an abstract ring isomorphism from $H^{2 *}(X)$ and $H^{*}\left(X^{G}\right)$ does not imply that $X$ is a conjugation manifold (see [16, Example 1]).

Proof. Obviously, (1) implies (2). Also, by Poincaré duality, the condition $H^{k}(X)=0$ for $0<k<2 n$ implies that $H^{\text {odd }}(X)=0$. 
Suppose that (2) holds true. Let $X_{0}$ obtained from $X$ by removing a small open $G$ invariant $4 n$-disk containing a fixed point. Then $X_{0}$ is a $G$-subspace with $X_{0}^{G}$ equal to $X^{G}$ minus an open $2 n$-disk. Hence,

$$
\operatorname{dim} H^{*}\left(X_{0}^{G}\right) \geq b_{0}\left(X_{0}^{G}\right)+b_{n}\left(X_{0}^{G}\right) \geq 1+b_{2 n}\left(X_{0}\right)=\operatorname{dim} H^{*}\left(X_{0}\right) .
$$

On the other hand, $\operatorname{dim} H^{*}\left(X_{0}^{G}\right) \leq \operatorname{dim} H^{*}\left(X_{0}\right)$ by Smith theory (see [2, Corollary 1.3.8]). Therefore, all the inequalities occuring in (2.15) are equalities, implying $b_{0}\left(X^{G}\right)=1$, $b_{n}\left(X_{0}^{G}\right)=b_{2 n}\left(X_{0}\right)$ and $H^{k}\left(X_{0}^{G}\right)=0$ for $0<k<n$. Also, by Proposition 2.4, $X_{0}$ is equivariantly formal and

$$
H_{G}^{2 n}\left(X_{0}^{G}\right)=H^{n}\left(X_{0}^{G}\right) u^{n} \oplus \mathbb{Z}_{2} u^{2 n} .
$$

Choose a section $\sigma: H^{2 n}\left(X_{0}\right) \rightarrow H_{G}^{2 n}\left(X_{0}\right)$ of $\rho: H_{G}^{2 n}\left(X_{0}\right) \rightarrow H^{2 n}\left(X_{0}\right)$. Let $\phi: H^{2 n}\left(X_{0}\right) \rightarrow$ $\mathbb{Z}_{2}$ be defined by letting $\phi(b)$ denote the coefficient of $u^{2 n}$ in $(r \circ \sigma)(b)$. Then by changing $\sigma(b)$ into $\sigma^{\prime}(b)=\sigma(b)+\phi(b) u^{2 n}$, one may assume that the image $\operatorname{im}(r \circ \sigma) \subseteq H^{n}\left(X_{0}^{G}\right) u$. As $b_{2 n}\left(X_{0}\right)=b_{n}\left(X_{0}^{G}\right), r \circ \sigma(a)$ is of the form $\kappa(a) u$ for an isomorphism $\kappa: H^{2}\left(X_{0}\right) \rightarrow H^{1}\left(X_{0}^{G}\right)$. Hence $X_{0}$ is a conjugation space. Now, the closure of the small $4 n$-disk removed in $X$ is what is called a conjugation cell in [23, Section 5.1]. By [23, Prop. 5.1], attaching a conjugation cell (by a $G$-map) to a conjugation space produces a conjugation space. Therefore, $X$ is a conjugation space.

We now restrict our attention to conjugation 4-manifolds. Here, the $G$-action preserves the orientation (as can be seen on the tangent space to a fixed point). In the statement below, $\mathbb{Z}_{(2)}$ denotes $\mathbb{Z}$ localized at 2 , the smallest subring of $\mathbb{Q}$ where all odd primes are invertible.

Proposition 2.16. Let $X$ be a smooth $G$-manifold of dimension 4 with $H_{1}\left(X ; \mathbb{Z}_{(2)}\right)=0$. Then, $X$ is a conjugation 4-manifold if and only if $X^{G} \neq \emptyset$ and $G$ acts on $H^{2}\left(X ; \mathbb{Z}_{(2)}\right)$ as multiplication by -1 .

Remark 2.17. The condition $H_{1}\left(X ; \mathbb{Z}_{(2)}\right)=0$ is equivalent to $H^{1}\left(X ; \mathbb{Z}_{2}\right)=0$. The condition on $H^{2}\left(X ; \mathbb{Z}_{(2)}\right)$ is then equivalent to $\tau$ acting as multiplication by -1 on $H^{*}(X ; \mathbb{Z})$ modulo torsion.

Proof. If $X$ is simply-connected with $G$ acting as $\tau_{*}=-1$ on $H^{2}(X ; \mathbb{Z})$, then $X$ is a conjugation manifold by results of V. Puppe (see [41, Theorem 5 and Remark 2]). We note that the same arguments (which are all 2-local) prove that $X$ is a conjugation 4manifold under our weaker assumption. Again, if $X$ were simply-connected then the other direction would follow from results of A. Edmonds [11, 2.4]. We leave the reader to verify our claim that the arguments of [11, 2.1-2.4] are 2-local, and again hold under our weaker assumption. It follows that the number of $\mathbb{Z}_{(2)}$-summands in $H^{2}\left(X ; \mathbb{Z}_{(2)}\right)$ on which $\tau_{*}=-1$ is equal to the rank of $H^{1}\left(\Sigma ; \mathbb{Z}_{2}\right)$, which equals the rank of $H^{2}\left(X ; \mathbb{Z}_{2}\right)$. Hence we have $\tau_{*}=-1$ on all of $H^{2}\left(X ; \mathbb{Z}_{(2)}\right)$.

Remark 2.18. If $X^{G}$ is orientable then its integral fundamental class $\left[X^{G}\right]$ represents a $\tau_{*}$-fixed class in $H_{2}(X ; \mathbb{Z})$ modulo odd torsion. But $\tau_{*}=-1$ on this quotient, so $X^{G}$ is null-homologous mod 2 in $X$. On the other hand, if $X^{G}$ is non-orientable then $w_{2}(X) \neq 0$ by Corollary 2.13 and, by [11, Cor. 5.2], the mod 2 homology class of the fixed set $X^{G}$ 
represents the Poincaré dual of $w_{2}(X)$. We conclude that $X^{G}$ is null-homologous mod 2 in $X$ if and only if $X^{G}$ is orientable, or equivalently if and only if $X$ is spin.

Proposition 2.19. Let $X$ be a simply connected smooth conjugation 4-manifold. Then $X$ is (non-equivariantly) homeomorphic to a connected sum of copies of $S^{2} \times S^{2}$, if $X$ is spin, or copies of $\mathbb{C} P^{2}$ and $\overline{\mathbb{C P}}^{2}$, if $X$ is non-spin.

Proof. In the non-spin case, if $X$ has a (positive) definite intersection form then $X$ is homeomorphic to a connected sum of copies of $\mathbb{C} P^{2}$ (by Donaldson [8] and Freedman [17]). If $X$ has an indefinite intersection form then $X$ is homeomorphic to a connected sum of $\mathbb{C} P^{2}$ 's and $\overline{\mathbb{C} P}^{2}$ 's by Freedman's Theorem and the classification of odd unimodular indefinite forms.

If $X$ is spin, we use the equivariant Hirzebruch formula [24, Formula (6)], [4, Prop. 6.15]:

$$
\operatorname{sign}(X, \tau)=\operatorname{sign}\left(\left(X^{\tau}\right)^{2}\right)
$$

where the right-hand side is given by evaluating the twisted Euler class of the normal bundle of $\Sigma$ in $X$. By Proposition 2.16, $G$ acts on $H^{2}(X ; \mathbb{Z})$ by multiplication by -1 . Therefore, $\operatorname{sign}(X)=-\operatorname{sign}(X, \tau)$. As $X$ is spin, the manifold $X^{G}$ is orientable, by Corollary 2.13, By Remark 2.18, the integral homology class represented by $X^{G}$ is zero, and hence $\operatorname{sign}(X)=0$. We deduce that the (even) integral intersection form of $X$ is a sum of hyperbolic forms and apply Freedman's theorem again.

Remark 2.20. The results of Proposition 2.16 and Remark 2.18 hold also for topological conjugation 4-manifolds, if the involution is assumed to be locally linear (see Section 6). The corresponding result to Proposition 2.19 is true in the spin topological locallylinear case: note that the index formula holds in this context [45, 14B] and the KirbySiebenmann invariant vanishes because $X$ is spin with $\operatorname{sign}(X)=0$. In the non-spin case, we don't know what happens if $X$ has a definite intersection form.

\section{The proof of Theorem A}

We divide the two directions of the proof into separate lemmas.

Lemma 3.1. Let $X$ be a connected, oriented, 4-dimensional conjugation manifold. Then $\left(X / G, X^{G}\right)$ is a $\mathbb{Z}_{2}$-knot.

Proof. As $X$ is a 4-dimensional conjugation manifold, the fixed point set $X^{G}$ is a closed connected surface which we call $\Sigma$. Let $V$ be a closed $G$-invariant tubular neighbourhood of $\Sigma$ in $X$ and let $K$ be the complement of the interior of $V$. Now, $M=X / G$ is a smooth manifold by Lemma 7.5. As noted in the introduction, $\tau$ preserves the orientation, so $M$ inherits an orientation and the projection map $p: X \rightarrow M$ is smooth, of degree 2 . We identify $\Sigma$ with $p(\Sigma)$. Then $\bar{V}=p(V)$ is a tubular neighbourhood of $\Sigma$ in $M$. One has $M=\bar{V} \cup \bar{K}$, with $\bar{K}=p(K)$. We have to prove that $M$ is a $\mathbb{Z}_{2}$-homology sphere.

As $X$ is a conjugation manifold, the restriction map $H_{G}^{*}(X) \rightarrow H_{G}^{*}\left(X^{G}\right)$ is injective by Proposition 2.4 and Remark 2.5. Also, $H_{G}^{*}(K) \approx H^{*}(\bar{K})$ and $H_{G}^{*}(\partial V) \approx H^{*}(\partial \bar{V})$ 
since the $G$-action on $K$ and $\partial V$ is free. The Mayer-Vietoris sequence in equivariant cohomology looks then like

$$
0 \rightarrow H_{G}^{*}(X) \rightarrow H_{G}^{*}(\Sigma) \oplus H^{*}(\bar{K}) \rightarrow H^{*}(\partial \bar{V}) \rightarrow 0 .
$$

Since $\Sigma \subset X$ is codimension 2 , the manifold $K$ is connected. Therefore, $\bar{K}$ has a nontrivial 2-fold cover, which implies that $b_{1}(K) \geq 1$. Also, since $X$ is a conjugation space, $H^{1}(X)=0$, so $\operatorname{dim} H_{G}^{1}(X)=1$. As $G$ acts trivially on $\Sigma$, one has $\operatorname{dim} H_{G}^{1}(\Sigma)=b_{1}(\Sigma)+1$. On the other hand, $b_{1}(\partial \bar{V}) \leq b_{1}(\Sigma)+1$ by the Gysin sequence of the circle bundle $\partial \bar{V} \rightarrow \Sigma$. Thus, Sequence (3.2) implies that $b_{1}(\bar{K})=1, b_{1}(\partial \bar{V})=b_{1}(\Sigma)+1$ and that $H^{*}(\Sigma) \oplus H^{*}(\bar{K}) \stackrel{\approx}{\longrightarrow} H^{*}(\partial \bar{V})$ is an isomorphism. This isomorphism sits in the MayerVietoris sequence for $M$ which implies that $H^{1}(M) \approx H^{3}(M)=0$. The decompositions $X=V \cup K$ and $M=\bar{V} \cup \bar{K}$ give the system of equations

$$
\left\{\begin{array}{l}
\chi(X)=\chi(\Sigma)+\chi(K)-\chi(\partial V) \\
\chi(M)=\chi(\Sigma)+\chi(\bar{K})-\chi(\partial \bar{V})
\end{array} .\right.
$$

As $\chi(\partial V)=2 \chi(\partial \bar{V})$ and $\chi(K)=2 \chi(\bar{K})$, one deduces that

$$
\chi(X)+\chi(\Sigma)=2 \chi(M),
$$

(compare [5, Chapter III, Theorem 7.10]). In our case, Equation (3.3) amounts to

$$
2+b_{2}(X)+2-b_{1}(\Sigma)=2 \chi(M)
$$

which implies that $\chi(M)=2$. Hence, $M$ is a $\mathbb{Z}_{2}$-homology sphere.

We now construct the correspondence from $\mathbb{Z}_{2}$-knots to conjugation manifolds.

Lemma 3.4. Let $(M, \Sigma)$ be a $\mathbb{Z}_{2}$-knot. Then $M$ has a unique oriented branched covering $\widehat{M} \rightarrow M$, with branched locus $\Sigma$. Moreover, $\widehat{M}$ is a conjugation manifold.

Proof. Let $W$ be a closed tubular neighbourhood of $\Sigma$ in $M$ and let $L$ be the complement of the interior of $W$. For $i=1,2$, one has the "Alexander duality"

$$
H^{i}(L) \stackrel{\approx}{\longrightarrow} H^{i+1}(M, L) \stackrel{\approx}{\longrightarrow} H^{i+1}(W, \partial W) \stackrel{\approx}{\longrightarrow} H^{i-1}(\Sigma),
$$

the last arrow being the Thom isomorphism. Thus, $b_{1}(L)=1, b_{2}(L)=b_{1}(\Sigma)$. Also, the Mayer-Vietoris exact sequence for the decomposition $M=W \cup L$ gives the isomorphisms

$$
H^{i}(\Sigma) \oplus H^{i}(L) \stackrel{\approx}{\longrightarrow} H^{i}(\partial W) \quad(i=1,2) .
$$

Therefore, $b_{1}(\partial W)=b_{2}(\partial W)=b_{1}(\Sigma)+1$. Since $\Sigma$ is of codimension 2 in $M$, the manifold $L$ is connected. As $b_{1}(L)=1$, there is a unique connected 2 -fold cover $\widetilde{L} \rightarrow L$. The induced cover $\widetilde{\partial W} \rightarrow \partial W$ is connected: otherwise, using Lemma 7.18 and Remark $7.22, \widetilde{L} \rightarrow L$ could be extended to a connected 2-fold covering of $M$, contradicting the assumption that $H^{1}(M)=0$. Hence, $\widetilde{L} \rightarrow L$ extends to a unique branched covering $\widehat{M} \rightarrow M$ with branched locus $\Sigma$, see Lemma 7.18. The complement $\widehat{W}$ of the interior of $\widetilde{L}$ is a tubular neighbourhood of $\Sigma$ in $\widehat{M}$.

In equivariant cohomology, the Mayer-Vietoris exact sequence for $\widehat{M}=\widehat{W} \cup \widetilde{L}$ starts as

$$
0 \rightarrow H_{G}^{1}(\widehat{M}) \rightarrow H_{G}^{1}(\Sigma) \oplus H^{1}(L) \rightarrow H^{1}(\partial W)
$$


with $H_{G}^{1}(\Sigma)=H^{1}(\Sigma) \oplus \mathbb{Z}_{2}$. Using the isomorphism of (3.5) for $i=1$, we deduce that $H_{G}^{1}(\widehat{M})=\mathbb{Z}_{2}$. This implies that $H^{1}(\widehat{M})=0$. Indeed, the choice of a $G$-fixed point in $\widehat{M}$ provides a section to the fibration $\widehat{M} \rightarrow \widehat{M}_{G} \rightarrow B G$. The homomorphism $H^{*}(B G) \rightarrow$ $H_{G}^{*}(\widehat{M})$ is then injective and the Serre spectral sequence for the fibration $\widehat{M} \rightarrow \widehat{M}_{G} \rightarrow B G$ gives the exact sequence

$$
0 \rightarrow H^{1}(B G) \rightarrow H_{G}^{1}(\widehat{M}) \rightarrow H^{0}\left(B G ; H^{1}(\widehat{M})\right) \rightarrow 0
$$

Thus, if $H_{G}^{1}(\widehat{M})=\mathbb{Z}_{2}$, then $0=H^{0}\left(B G ; H^{1}(\widehat{M})\right)=H^{1}(\widehat{M})^{G}$. But, a finite dimensional $G$-vector space $V$ over $\mathbb{Z}_{2}$ vanishes if $V^{G}=0\left(\right.$ if $0 \neq v \neq \tau(v)$ then $\left.0 \neq v+\tau(v) \in V^{G}\right)$. Therefore, $H^{1}(\widehat{M})=0$. By Poincaré duality, we have then $H^{\text {odd }}(\widehat{M})=0$. Equation (3.3) holds with the same proof and gives $\chi(\widehat{M})+\chi(\Sigma)=2 \chi(M)=4$ which implies that $b_{2}(\widehat{M})=b_{1}(\sigma)$. Therefore, $\widehat{M}$ is a conjugation manifold by Proposition 2.15 .

The proof of Theorem A. With the equivalence relations used for the statement of Theorem $\mathrm{A}$, let $\mathcal{C}$ be the set of equivalence classes of oriented conjugation 4-manifolds and let $\mathcal{N}$ be that of classes of $\mathbb{Z}_{2}$-knots. By Lemma 3.1, the correspondence $X \mapsto X / G$ associates a a $\mathbb{Z}_{2}$-knot to a conjugation 4 -manifold. By Lemma 7.13, this correspondence produces a well defined map $\Phi: \mathcal{C} \rightarrow \mathcal{N}$.

By Lemma 3.4, the correspondence $M \mapsto \widehat{M}$ sends a $\mathbb{Z}_{2}$-knot to an oriented conjugation 4-manifold. By Lemma 7.23, this provides a well defined map $\Psi: \mathcal{N} \rightarrow \mathcal{C}$.

The fact that $\Phi \circ \Psi=\mathrm{id}_{\mathcal{N}}$ is guaranteed by Lemma 7.17. That $\Psi \circ \Phi=\mathrm{id}_{\mathcal{C}}$ follows from Lemma 7.5 (since $p: X \rightarrow X / G$ is a branched covering with branched locus $\Sigma$ ), and the uniqueness part of Lemma 7.18 .

\section{ExAmples And REMARKS}

We present some examples and remarks concerning the bijection of Theorem A.

Example 4.1. Let $\Sigma=S^{2} \subset \mathbb{R}^{3} \times 0 \subset \mathbb{R}^{3} \times \mathbb{C}$. The involution on $X=S^{4} \subset \mathbb{R}^{3} \times \mathbb{C}$ induced by the linear map $(w, z) \mapsto(w,-z)$ is a conjugation by Proposition 2.6. The map $q: \mathbb{R}^{3} \times \mathbb{C} \rightarrow \mathbb{R}^{3} \times \mathbb{C}$ given by $q(w, z)=\left(w, z^{2}\right)$ identifies $\left(\mathbb{R}^{3} \times \mathbb{C}\right) / G$ with $\mathbb{R}^{3} \times \mathbb{C}$ and $S^{4} / G$ with $S^{4}$. This shows that, with the smooth structure of Lemma 7.5, $S^{4} / G$ is diffeomorphic to $S^{4}$. The image in $X / G$ of the sphere $S^{3} \subset \mathbb{R}^{3} \times \mathbb{R}$ is a 3-disk with boundary $\Sigma$. Hence, under the bijection $X \mapsto X / G$ of Theorem A, the standard conjugation sphere corresponds to the trivial knot.

Interesting examples occur with $\Sigma=\mathbb{R} P^{2}$.

Proposition 4.2. Let $(M, \Sigma)$ be a $\mathbb{Z}_{2}$-knot with $\Sigma=\mathbb{R} P^{2}$. Then, any homeomorphism $h:(M, \Sigma) \rightarrow(M, \Sigma)$ is of degree one. In consequence, $(M, \sigma)$ and $(-M, \sigma)$ are inequivalent $\mathbb{Z}_{2}$-knots.

Proof. By the uniqueness of branched coverings [31, Prop. 3], $h$ is covered by a homeomorphism $\widehat{h}: \widehat{M} \rightarrow \widehat{M}$. The cohomology ring $H^{*}(\widehat{M})$ is isomorphic to that of $\mathbb{C} P^{2}$. By the universal coefficient theorem and Poincaré duality, $H^{*}(\widehat{M} ; \mathbb{Q}) \approx \mathbb{Q}[a] /\left(a^{3}\right)$, with 
$a \in H^{2}(\widehat{M} ; \mathbb{Q})$. Thus, $\widehat{h}^{*}(a)=\lambda a$ for some $\lambda \in \mathbb{Q}$ and $\widehat{h}\left(a^{2}\right)=\lambda^{2} a^{2}$. Therefore, $\widehat{h}$ is of degree 1 and so is $h$.

Example 4.3. The projective space $X=\mathbb{C} P^{2}$ with the complex conjugation is a conjugation manifold with $X^{G}=\mathbb{R} P^{2}$. The quotient $X / G$ is diffeomorphic to $S^{4}$ (see Proposition 5.4 below), and $\left(X / G, \mathbb{R} P^{2}\right)$ is the "standard" embedding of $\mathbb{R} P^{2}$ into $S^{4}$ (see Lawson [29] for an explicit description). Let $r: X \rightarrow X$ be the diffeomorphism given by $r(x, y, z)=(x, y,-z)$. It commutes with the complex conjugation and thus descends to an involution $\bar{r}$ of $X / G \approx S^{4}$, preserving $\mathbb{R} P^{2}$. The diffeomorphism $r$ is isotopic to the identity by the isotopy $r_{t}(x, y, z)=\left(x, y, e^{i \pi t} z\right)$. Thus, $r$ and $\bar{r}$ are of degree 1 , in accordance with Proposition 4.2.

\section{Applications to knots in $S^{4}$}

The 2-fold branched coverings in which the branch locus is a knotted 2-sphere in $S^{4}$, are particularly interesting, We first investigate the relation between the fundamental groups of a conjugation 4-manifold $X$ and its quotient $X / G$.

Let $(M, \Sigma)$ be a $\mathbb{Z}_{2}$-knot, and let $W$ be a closed tubular neighbourhood of $\Sigma$. Then $\partial W \rightarrow \Sigma$ is a locally trivial $S^{1}$-bundle. We get an exact sequence

$$
C_{\infty} \rightarrow \pi_{1}(\partial W) \rightarrow \pi_{1}(\Sigma) \rightarrow 1
$$

where $C_{\infty}$ is an infinite cyclic group. The image of a generator $\gamma \in C_{\infty}$ via the composed homomorphism $C_{\infty} \rightarrow \pi_{1}(\partial W) \rightarrow \pi_{1}(M-\Sigma)$ is called a meridian, and denoted $m \in$ $\pi_{1}(M-\Sigma)$. A $\mathbb{Z}_{2}$-knot admits two meridians, which are inverses of each other. From the proof of Lemma 3.4 we see that $H^{1}(M-\Sigma) \cong \mathbb{Z}_{2}$, so there is a unique epimorphism $\phi: \pi_{1}(M-\Sigma) \rightarrow \mathbb{Z}_{2}$. Furthermore, $\phi(m) \neq 0$.

Proposition 5.1. Let $(M, \Sigma)$ be a $\mathbb{Z}_{2}$-knot and let $\widehat{M}$ be the associated conjugation 4manifold. If $m \in \pi_{1}(M-\Sigma)$ is a meridian, then

$$
\pi_{1}(\widehat{M}) \cong \operatorname{ker} \phi /\left\langle m^{2}\right\rangle
$$

Proof. Let $M=W \cup L$, where $L \simeq M-\Sigma$, and similarly $\widehat{M}=\widehat{W} \cup \widetilde{L}$. As noted above, $\pi_{1}(\partial W) \rightarrow \pi_{1}(\Sigma)$ is surjective, and so is $\pi_{1}(\widetilde{\partial W}) \rightarrow \pi_{1}(\Sigma)$ by the same argument. We also have the description $\pi_{1}(\widetilde{L})=\operatorname{ker} \phi$. Let $\gamma$ be a generator of $C_{\infty}$ sent to $m \in \pi_{1}(L)$ and let $\bar{\gamma}$ be the image of $\gamma$ in $\operatorname{ker}\left(\pi_{1}(\partial W) \rightarrow \pi_{1}(\Sigma)\right)$. Then $\operatorname{ker}\left(\pi_{1}(\widetilde{\partial W}) \rightarrow \pi_{1}(\Sigma)\right)$ is generated by $\gamma^{2}$. Proposition 5.1 follows from the Van Kampen theorem, because of the surjectivity of the map $\pi_{1}(\widetilde{\partial W}) \rightarrow \pi_{1}(\Sigma)$.

Corollary 5.2. Let $(M, \Sigma)$ be a $\mathbb{Z}_{2}$-knot and let $m \in \pi_{1}(M-\Sigma)$ be a meridian. The associated conjugation 4-manifold $\widehat{M}$ is simply connected if and only if ker $\phi$ is the normal closure of $m^{2}$.

Proposition 5.3. Let $X$ be a conjugation 4-manifold. If $X$ is simply connected, then $X / G$ is homeomorphic to $S^{4}$. 
Proof. Let $M=X / G$ and $\Sigma=X^{G}$. We know that $(M, \Sigma)$ is a $\mathbb{Z}_{2}$-knot. Let $m$ be a meridian for $(M, \Sigma)$. By Corollary [5.2, $\operatorname{ker} \phi$ is the normal closure of $m^{2}$. But $\phi(m) \neq 0$, as seen in the proof of Lemma 3.4 since the 2 -fold covering given by $\phi$ is not trivial over $\partial W$. This implies that $\pi_{1}(M-\Sigma)$ is the normal closure of $m$. As above, the Van Kampen theorem implies that $M$ is simply connected. By the universal coefficient theorem and Poincaré duality, a simply connected $\mathbb{Z}_{2}$-homology sphere is an integral homology sphere. Therefore, $M$ is homotopy equivalent to $S^{4}$, and hence homeomorphic to $S^{4}$ by Freedman's proof [17] of the Poincaré conjecture in dimension 4.

As mentioned in the Introduction, it is well-known that the classical conjugation 4manifolds all have quotient the standard smooth $S^{4}$. For the reader's convenience, we include a proof using results on group actions due to Bredon and Orlik-Raymond (but not the deep results of Freedman [17] or Cerf [6]).

Proposition 5.4 (Arnold, Kuiper, Massey). Let $(X, G)$ denote the classical conjugation 4-manifolds (i) $\mathbb{C} P^{2}$ with complex conjugation, or (ii) $S^{2} \times S^{2}$ with the complex conjugation in each factor. Then the quotient $X / G$ with the smooth structure given by Lemma 7.5 is diffeomorphic to the standard smooth $S^{4}$.

Proof. There is a smooth $S O(3)$-action on $X=\mathbb{C} P^{2}$ which commutes with complex conjugation. By Lemma 7.14, the quotient space $X / G$ inherits the structure of a smooth $S O(3)$-manifold. The classification of smooth cohomogeneity one actions of $S O(3)$ by Bredon [5, Theorem VI.6.3] shows that $(X / G, S O(3))$ is $S O(3)$-equivariantly diffeomorphic to the standard $S O(3)$-action on $S^{4}$. Therefore $X / G$ is diffeomorphic to $S^{4}$. The case $X=S^{2} \times S^{2}$ is done in Example 7.8 and in Example 7.15 .

To describe other examples for Proposition 5.3, we start with a technique of Mazur and Zeeman [47]. Let $N$ be a smooth oriented 3-dimensional $\mathbb{Z}_{2}$-homology sphere. Let $h: N \rightarrow N$ be a diffeomorphism such that

(1) $h$ preserves the orientation.

(2) $h$ has a fixed point.

Let $T_{h}=N \times[0,1] /\{(x, 1) \sim(h(x), 0)\}$ be the mapping torus of $h$. Choose a fixed point $x_{0} \in N$ for $h$. The map $\psi_{0}:[0,1] \rightarrow T_{h}$ given by $\psi_{0}(t)=\left[x_{0}, t\right]$ is a parametrisation of a circle $S$ in $T_{h}$. The normal bundle of $S$ is trivial by condition (1). We can then choose a parametrisation $\psi:[0,1] \times D^{3} \rightarrow T_{h}$ of a tubular neighbourhood of $S$ extending $\psi_{0}$. We consider the the surgery using $\psi$, producing a smooth 4-manifold

$$
M_{h, \psi}=\left[T_{h}-\psi\left([0,1] \times \operatorname{int} D^{3}\right)\right] \cup_{\dot{\psi}} D^{2} \times S^{2},
$$

where $\dot{\psi}$ is the restriction of $\psi$ to $[0,1] \times S^{2}$. The manifold $M_{h, \psi}$ contains $\{0\} \times S^{2}$, so we get a pair $\left(M_{h, \psi}, S^{2}\right)$.

\section{Lemma 5.6.}

(1) $\left(M_{h, \psi}, S^{2}\right)$ is a $\mathbb{Z}_{2}$-knot.

(2) If $N$ is a $\mathbb{Z}$-homology sphere, then $M_{h, \psi}$ is a $\mathbb{Z}$-homology sphere.

(3) The fundamental group of $M_{h, \psi}$ is isomorphic to the quotient of $\pi_{1}(N)$ by the relations $x=h_{*}(X)$ for all $x \in \pi_{1}(N)$. 
(4) The fundamental group of the associated conjugation 4-manifold $\widehat{M}_{h, \psi}$ is isomorphic to the quotient of $\pi_{1}(N)$ by the relations $x=h_{*}^{2}(X)$ for all $x \in \pi_{1}(N)$, where $h^{2}=h \circ h$.

Proof. By the Serre spectral sequence of the bundle $N \rightarrow T_{h} \rightarrow S^{1}$, the homology of $T_{h}$ is isomorphic to that of $N \times S^{1}$. Conclusions (1) and (2) then follow from the Mayer-Vietoris sequence of the decomposition (5.5).

For the fundamental group, choose a base point $\tilde{y}_{0} \in S^{2}$ and let $y_{0}=\dot{\psi}\left(\tilde{y}_{0}, 0\right) \in N \subset T_{h}$. The map $\psi_{1}(t)=\dot{\psi}\left(\tilde{y}_{0}, t\right)$ represents an element $m \in \pi_{1}\left(T_{h}, y_{0}\right)$ and the fundamental group of $T_{h}$ is the HNN-extension

$$
\pi_{1}\left(T_{h}, y_{0}\right) \approx\left\langle\pi_{1}(N), m ; m x m^{-1}=h_{*}(x), x \in \pi_{1}(N)\right\rangle
$$

(we use the notations of [32, $\S$ IV.2]). By the Van Kampen theorem applied to the decomposition (5.5), $\pi_{1}\left(M_{h, \psi}, y_{0}\right)$ is the quotient of $\pi_{1}\left(T_{h}, y_{0}\right)$ by the normal closure of $m$. This proves $(3)$.

To prove (4), let $L=M_{h, \psi}-\left(\operatorname{int} D^{2} \times S^{2}\right)=T_{h}-\psi\left([0,1] \times \operatorname{int} D^{3}\right)$. The element $m$ is a meridian and the epimorphism $\phi: \pi_{1}\left(M_{h, \psi}-S^{2}, y_{0}\right) \rightarrow \mathbb{Z}_{2}$ sends $m$ to the generator and $\pi_{1}(N)$ to 0 . Thus, $\widetilde{L}$ is the mapping torus of $h^{2}$ and there is a decomposition

$$
\widehat{M}_{h, \psi}=M_{h^{2}, \hat{\psi}}=T_{h^{2}} \cup D^{2} \times S^{2}
$$

for some parametrisation $\hat{\psi}$ analogous to (5.5). Conclusion (4) follows from Van Kampen's theorem.

Examples of 3-dimensional $\mathbb{Z}_{2}$-homology spheres are given by cyclic branched coverings of classical knots. In such case, following results of Zeeman [47, Corollary 1, p. 486] and Gordon [21, Theorem 3.1], Pao proved the following result (see [40, $\S 3]$ ).

Lemma 5.8. Let $N$ be the p-fold cyclic covering $S^{3}$ branched over a knot. Let $h: N \rightarrow N$ be the diffeomorphism corresponding to the action of a generator of $\mathbb{Z}_{p}$. Then $M_{h, \psi}$ is diffeomorphic to $S^{4}$ for any $\psi$.

Example 5.9. Let $N=L(p, q)$ be a 3-dimensional lens space with $p$ odd. By [42, Satz 6], $N$ is the 2-fold branched covering of $S^{3}$ for some knot. By Lemma 5.8, this gives an involution $h$ of $N$ for which $M_{h, \psi}$ is diffeomorphic to $S^{4}$ (but $\pi_{1}\left(\widehat{M}_{h, \psi}\right) \approx \mathbb{Z}_{p}$ ).

Example 5.10. Let $N$ be the Poincaré homology sphere

$$
N=S O(3) / A_{5}
$$

with $\pi_{1}(N)=\Delta$ the binary icosahedral group, defined as the universal central extension of $A_{5}$. The conjugation in $S O(3)$ by an element $b \in A_{5}$ produces a diffeomorphism $h: N \rightarrow N$. The induced automorphism $h_{*}$ of $\Delta$ is the conjugation by an element $\tilde{b}$ over b. Lemma 5.6 has the following consequences on the manifolds $M_{h, \psi}$ and $\widehat{M}_{h, \psi}$ (for any choice of $\psi$ ).

- $M_{h, \psi}$ is an integral homology sphere.

- If $b$ is not trivial, $M_{h, \psi}$ is a homotopy sphere. 
- If $b^{2}$ is not trivial, $\widehat{M}_{h, \psi}$ is a homotopy sphere. Indeed, the relations $x=b^{2} x b^{-2}$ kills $A_{5}$ which is simple, so the relations $y=\tilde{b}^{2} y \tilde{b}^{-2}$ kills $\Delta$.

It is classical that $N=S O(3) / A_{5}$ is the 5-fold covering of $S^{3}$ branched over the trefoil knot [43, $\S 65]$. By Lemma 5.8 and (5.7), $M_{h, \psi}$ and $\widehat{M}_{h, \psi}$ are both diffeomorphic to $S^{4}$ for some choice of $b$ of order 5 .

Remark 5.11. Example 5.10 with $b^{2} \neq 1$ produces counter-examples to the generalised Smith conjecture in dimension 4: a smooth involution on a homotopy 4-sphere with a non-trivial knot (even topologically) as fixed point set. The first such examples appeared in 1966 in the work of Giffen [19, Theorem 3.3]. Later Gordon [20], [21] and Sumners [44] proved that there are infinitely many non-equivalent knots in $S^{4}$ which are the fixed point sets of smooth involutions. In addition, Cameron Gordon (email communication) has informed us that the quotient spaces $S^{4} / G$ of his examples are obtained from $S^{4}$ by a "Gluck surgery" construction on a (twist-spun) knot, and hence are diffeomorphic to $S^{4}$ by [21, Theorem 3.1]. Proposition 2.6 now implies the following statement:

Proposition 5.12. There are infinitely many smooth conjugations on $S^{4}$ which are topologically inequivalent.

Remark 5.13. Further examples of smooth conjugation 4-manifolds might also come from the rim surgery construction of Fintushel and Stern [14, [15], although it is not clear at present how to detect exotic smooth structures on the 2-fold branched coverings of $\mathbb{Z}_{2}$-homology 4 -spheres.

\section{Conjugations on topological 4-Manifolds}

We first state a topological version of Theorem A. The involution in a topological conjugation manifold is supposed to be locally linear (also called locally smooth in [5. Chapter IV]). We also consider topological locally flat $\mathbb{Z}_{2}$-knots. Two of them, $(M, \Sigma)$ and $\left(M^{\prime}, \Sigma^{\prime}\right)$ are topologically equivalent if there is an orientation-preserving homeomorphism $h: M \rightarrow M^{\prime}$ such that $h(\Sigma)=\Sigma^{\prime}$.

Theorem B. The correspondence $X \mapsto\left(X / G, X^{G}\right)$ defines a bijection between

(a) the orientation-preserving G-homeomorphism classes of oriented connected topological conjugation 4-manifolds, and

(b) the topological equivalence classes of topological locally flat $\mathbb{Z}_{2}$-knots.

Proof. The orbit space $X / G$ of a locally linear action is a closed topological 4-manifold, and the image $p\left(X^{G}\right)$ of the fixed set is a locally flat submanifold of $X / G$. By [18, $\S 9.3$, this submanifold admits a normal bundle and therefore a tubular neighbourhood. Lifting this tubular neighbourhood to $X$ gives a $G$-invariant tubular neighbourhood for $X^{G}$ in $X$. Similarly, a locally flat submanifold $\Sigma$ of $M$ admits a tubular neighbourhood. The proofs of Lemmas 3.1 and 3.4 can be carried out using these tubes (using Remark 2.5 as noted to justify the application of Proposition 2.4). The existence of the 2-fold branched covering $\widehat{M} \rightarrow M$ is guaranteed by [31, Prop. 2].

The arguments of the proof of Theorem A (end of Section 3) are much simpler than in the smooth case. They come from the fact that the constructions under consideration 
are functorial for homeomorphisms. For $X \mapsto X / G$, this is obvious. For $M \mapsto \widehat{M}$, this follows from [31, Prop. 3].

Examples 6.1. Finashin, Kreck and Viro [12] have constructed an infinite family of topologically equivalent, but smoothly inequivalent embeddings of $\Sigma=\#_{10} \mathbb{R} P^{2}$ in $S^{4}$. In all these examples, the fundamental group of the complement is just $\mathbb{Z} / 2$. By Theorem $A$, this gives an infinite family of smooth conjugation 4-manifolds which are topologically equivalent by Theorem B but not diffeomorphic (since the associated conjugation 4-manifolds are non-diffeomorphic Dolgachev surfaces).

Other examples, this time of topologically equivalent, but smoothly inequivalent knotted surfaces in $\mathbb{C} P^{2}$, were constructed by Finashin [13].

Remark 6.2. Non-oriented versions of Theorems A and B hold: just leave out the words "orientation preserving" and "oriented" in (a) and in the definition of $\mathbb{Z}_{2}$-knots. For instance, $(M, \Sigma)$ is equivalent to $(-M, \Sigma)$. This is definitely a coarser equivalence relation, as seen in Proposition 4.2.

\section{Appendix: Branched COVERings And Smooth StruCtures}

Branched covering spaces of manifolds is a classical topic in geometric topology, which appears frequently in the literature (for example, see the references cited in Durfee and Kauffman [9] and Lines [31]). By a 2-fold branched covering we mean a ramified 2-fold covering in which the branch locus is a closed submanifold of codimension two.

In the proof of Theorem A, we need to know the relationship between smooth structures on the total space and quotient space of a 2 -fold branched covering of 4-manifolds. This material may be well-known, but we were not able to find the right references for our proofs. Smooth manifolds are so strikingly different from topological manifolds in dimension 4, that these issues perhaps deserve some extra attention. This section is included to provide a detailed account, as a service to the reader.

The map $z \mapsto z^{2}$ from $\mathbb{C}$ to $\mathbb{C}$ is the simplest example of a 2-fold branched covering, with $\{0\}$ being the (strict) branched locus. We will need a precise local description of this example. Let $D$ be the unit disk in $\mathbb{C}$. Identify $S O(2)$ with $S^{1}$ and $O(2)$ with the $\mathbb{R}$-linear isometries of $\mathbb{C}$. The homomorphism $\gamma \mapsto \gamma^{2}$ of $S^{1}$ extends to a smooth epimorphism

$$
\psi: O(2) \rightarrow O(2) \text {. }
$$

Let $P \rightarrow K$ be a smooth principal $O(2)$-bundle. Consider the two Borel constructions

$$
P \times_{O(2)} D=P \times D /\{(a \alpha, z)=(a, \alpha z) \mid \alpha \in O(2)\}
$$

and

$$
P \overline{\times}_{O(2)} D=P \times D /\{(a \alpha, z)=(a, \psi(\alpha) z) \mid \alpha \in O(2)\} .
$$

The map $(a, z) \mapsto\left(a, z^{2}\right)$ descends to a smooth surjection

$$
q: P \times_{O(2)} D \rightarrow P \overline{\times}_{O(2)} D
$$

which will be our local model for a 2-fold branched covering with branched locus $K$. The general definition is the following. 
Definition 7.3. Let $M$ be a smooth manifold of dimension $n$ with a codimension 2 submanifold $N$. A smooth map $p:(X, Y) \rightarrow(M, N)$ is a (2-fold) branched covering with branched locus $N$ if

- $p: X-Y \rightarrow M-N$ is a smooth 2-fold covering, in particular a local diffeomorphism.

- $p: Y \rightarrow N$ is a diffeomorphism. We often identify $Y$ with $N$ via $p$.

- there are closed tubular neighbourhoods $Y \subset \tilde{V} \subset X$ and $N \subset V \subset M$ for $Y$ and $N$ such that $p(\tilde{V})=V$ and $p \mid \tilde{V}$ has, up to diffeomorphism, the form of (7.2).

This definition may be compared with the properties of smooth branched coverings given in Durfee and Kauffman [9, Prop. 1.1]. On one hand, it is simpler because we are dealing with the special case of 2 -fold coverings. On the other hand, Definition 7.3 is more precise: we specify the model around the branched locus (compare part (ii) of Proposition 1.1 in [9]).

7.4. Quotient structure. Our first task is to show how a smooth $G$-action on a closed manifold $X$ determines a smooth structure on the quotient space $X / G$, which is unique up to diffeomorphism.

Lemma 7.5. Let $X$ be a smooth $G$-manifold such that the fixed point set $X^{G}$ is a closed manifold of codimension 2. Then,

(1) $X / G$ admits the structure of a smooth manifold such that $p: X \rightarrow X / G$ is a branched covering with branched locus $p\left(X^{G}\right)$.

(2) if $X$ is a closed manifold, any two such structures on $X / G$ are diffeomorphic.

Proof. Let $X_{\text {free }}=X-X^{G}$. The quotient map $p_{\text {free }}: X_{\text {free }} \rightarrow X_{\text {free }} / G$ is a covering projection, and hence $X_{\text {free }} / G$ has a unique a smooth structure such that $p_{\text {free }}$ is a local diffeomorphism. We shall put a smooth structure on a neighbourhood of $X^{G}$ in $X / G$ which agrees with that on $X_{\text {free }}$.

Step 1. Existence. To a $G$-invariant Riemannian metric $g$ on $X$, we will associate a smooth structure $(X / G)_{g}$ on $X / G$ satisfying condition (1) of Lemma 7.5, Let $\nu$ be the normal bundle to $X^{G}$ in $X$ given by the metric $g$, so $\nu_{x}=\left(T_{x} X^{G}\right)^{\perp} \subset T_{x} X$ for $x \in$ $X^{G}$. Let $P \rightarrow X^{G}$ be the $O(2)$-principal bundle associated to $\nu$, so $P_{x}$ is the space of orthonormal frames in $\nu_{x}$. The space $P \times_{O(2)} D$ is a smooth $G$-manifold, with the involution $\tau(a, z)=(a,-z)$. It is $G$-diffeomorphic to the unit disk bundle associated to $\nu$

Let us perform the equivariant tubular neighbourhood construction [5, Chapter VI, Theorem 2.2] using the exponential map for the metric $g$. We say that $g$ is calibrated around $X^{G}$ if the exponential map is an embedding on $P \times_{O(2)} D$. As $X^{G}$ is compact, one can multiply $g$ by a constant (scaling) so that it is calibrated. Therefore, there exists a $G$-invariant neighbourhood $V$ of $X^{G}$ in $X$ and a $G$-equivariant diffeomorphism

$$
\varphi: P \times_{O(2)} D \stackrel{\approx}{\longrightarrow} V .
$$


The map $q$ of (7.2) sits in a commutative diagram

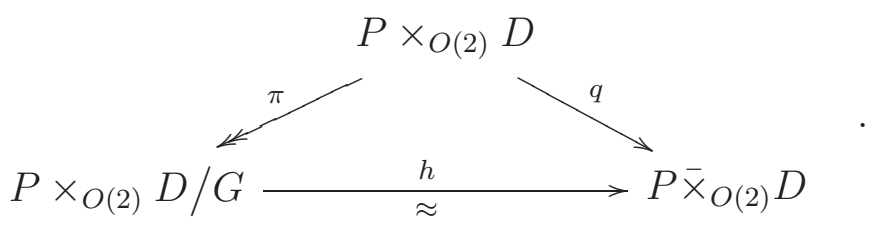

The map $h$ is a continuous bijection between compact spaces, and hence a homeomorphism. Therefore, we get a commutative diagram

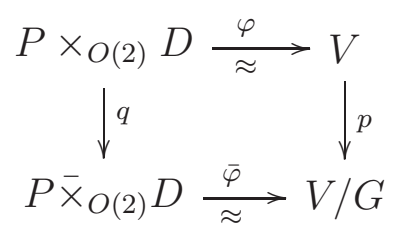

where $\bar{\varphi}$ is a homeomorphism. As $q$ is smooth, the homeomorphism $\bar{\varphi}$ provides a smooth structure on $V / G$, which is a neighbourhood of $X^{G}$ in $X / G$, and the projection $p: V \rightarrow$ $V / G$ is smooth. As $\varphi$ composed with the inclusion $V \hookrightarrow X$ is a smooth embedding, the smooth structures on $X_{\text {free }} / G$ and on $V / G$ agree on $V / G-X^{G}$. This defines the structure $(X / G)_{g}$, which does not depends on the scaling. By diagram (17.6), it satisfies condition (1) of Lemma 7.5 .

Step 2. Metric independence. If $g$ and $g^{\prime}$ are two $G$-equivariant Riemannian metrics on $X$, and if $X$ is a closed manifold, then we will show that $(X / G)_{g}$ and $(X / G)_{g^{\prime}}$ are diffeomorphic. The family $t g^{\prime}+(1-t) g(t \in[0,1])$ defines a $G$-invariant Riemannian metric $\check{g}$ on the manifold with boundary $L=X \times[0,1]$. By scaling $\check{g}$, we may suppose that it is calibrated around $L^{G}$. The above construction provides a smooth structure $(L / G)_{\check{g}}$ (the presence of boundaries does not create difficulties). Using diagram (7.6), one shows that the projection $b:(L / G)_{\breve{g}} \rightarrow[0,1]$ is a submersion. As $X / G$ is a closed manifold, integrating a gradient-like vector field for $b$ provides a diffeomorphism between $b^{-1}(0)=(X / G)_{g}$ and $b^{-1}(1)=(X / G)_{g^{\prime}}$.

Step 3. Uniqueness. Recall that all smooth structures on $X / G$ satisfying condition (1) of Lemma 7.5 will agree on $X / G-X^{G}$. We have to show that this is the case around $X^{G}$.

Suppose that $X / G$ is endowed with a smooth structure such that $p: X \rightarrow X / G$ is a 2-fold branched covering with branched locus $p\left(X^{G}\right)=X^{G}$. By definition, there is a neighbourhood $W$ of $X^{G}$ in $X$ and a commutative diagram

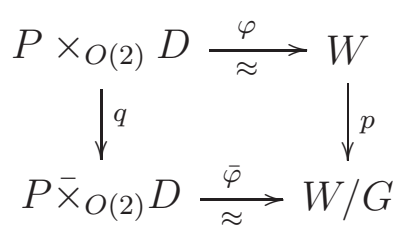

where $\varphi$ and $\bar{\varphi}$ are smooth embeddings. If we restrict these embeddings to $P \times_{O(2)} D^{*}$ and $P \overline{\times}_{O(2)} D^{*}$, where $D^{*}=D-\{0\}$, then diagram (7.7) is a morphism of 2-fold (unbranched) 
covering spaces. The deck transformation on $P \times_{O(2)} D^{*}$ is given by $(a, z) \mapsto(a,-z)$. Hence, $\varphi$ is a a $G$-equivariant embedding.

Endow $P \times D$ with a Riemannian metric which is the product of a $O(2)$-invariant Riemannian metric on $P$ with the standard metric on $D$. This descends to a Riemannian metric on $P \times_{O(2)} D$, which is $G$-invariant. One can construct a $G$-invariant Riemannian metric $g$ on $X$ so that $\varphi$ is an isometry. Hence, $\varphi$ is actually the normal exponential map and diagram (7.7) plays the role of diagram (17.6) to define the smooth structure $(X / G)_{g}$ around $X / G$, via the homeomorphism $\bar{\varphi}$. As $\bar{\varphi}$ is a smooth embedding, the given smooth structure on $X / G$ coincides with $(X / G)_{g}$.

Now, let $(X / G)^{\prime}$ and $(X / G)^{\prime \prime}$ be two smooth structures satisfying condition (1). By the above argument, there are $G$-invariant Riemannian metrics $g^{\prime}$ and $g^{\prime \prime}$ on $X$ such that $(X / G)^{\prime}=(X / G)_{g^{\prime}}$ and $(X / G)^{\prime \prime}=(X / G)_{g^{\prime \prime}}$. But $(X / G)_{g^{\prime}}$ and $(X / G)_{g^{\prime \prime}}$ are diffeomorphic, as seen in Step 2.

Example 7.8. Let us consider $S^{2} \subset \mathbb{C} \times \mathbb{R}$ with the involution $(z, t) \mapsto(z,-t)$ and let $X=S^{2} \times S^{2}$ endowed with the diagonal involution $\tau$. One can construct a smooth 2 -fold branched covering $\pi: X \rightarrow S^{4}$ by explicit formulas, which descends to a homeomorphism $\bar{\pi}: X / G \stackrel{\approx}{\longrightarrow} S^{4}$. We remark that an application of Lemma 7.5 then gives the well-known result that $X / G$, with the smooth structure of Lemma 7.5, is diffeomorphic to $S^{4}$.

Our coordinates on $X$ will be $\left(w_{1}, w_{2}\right)$, where $w_{j}=\left(r_{j} e^{i \theta_{j}}, t_{j}\right)$ for $j=1,2$. The standard $\left(S^{1} \times S^{1}\right)$-action on $X$ is defined by

$$
\left(z_{1}, z_{2}\right) \cdot\left(w_{1}, w_{2}\right)=\left(z_{1} r_{1} e^{i \theta_{1}}, t_{1}, z_{2} r_{2} e^{i \theta_{2}}, t_{2}\right)
$$

for all $\left(z_{1}, z_{2}\right) \in S^{1} \times S^{1}$. Let $\tilde{\pi}: X \rightarrow \mathbb{C} \times \mathbb{C} \times \mathbb{R}$ be defined by

$$
\tilde{\pi}\left(w_{1}, w_{2}\right)=\left(r_{1}^{2} e^{i \theta_{1}}, r_{2}^{2} e^{i \theta_{2}}, t_{1} t_{2}\right) .
$$

The function $L=L\left(w_{1}, w_{2}\right)=\left\|\tilde{\pi}\left(w_{1}, w_{2}\right)\right\|^{2}=r_{1}^{4}+r_{2}^{4}+\left(t_{1} t_{2}\right)^{2}$ never vanishes, so

$$
\pi\left(w_{1}, w_{2}\right)=\frac{1}{\sqrt{L}} \tilde{\pi}\left(w_{1}, w_{2}\right)
$$

defines a smooth map $\pi: X \rightarrow S^{4}$, which is $\left(S^{1} \times S^{1}\right)$-equivariant. One checks that $\pi$ descends to a homeomorphism $\bar{\pi}: X / G \stackrel{\approx}{\longrightarrow} S^{4}$.

It remains to show that $\pi: X \rightarrow S^{4}$ is a branched covering. Let us consider the following diagram

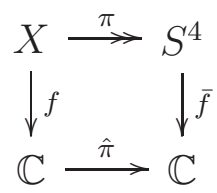

where

$$
f\left(w_{1}, w_{2}\right)=\frac{1}{\sqrt[4]{L}}\left(t_{1}+i t_{2}\right) \quad, \quad \bar{f}\left(\rho_{1} e^{i \theta_{1}}, \rho_{2} e^{i \theta_{2}}, t\right)=\rho_{2}-\rho_{1}+2 i t
$$

and $\hat{\pi}(z)=z^{2}$. As $r_{j}^{2}+t_{j}^{2}=1$, one has $t_{1}^{2}-t_{2}^{2}=r_{2}^{2}-r_{1}^{2}$ and diagram (17.10) commutes. Observe that $f$ and $\bar{f}$ are $\left(S^{1} \times S^{1}\right)$-invariant. 
Derivative computations show that $0 \in \mathbb{C}$ is a regular value for $f$. This produces a $\left(S^{1} \times S^{1}\right)$-invariant trivialization of the normal bundle to $X^{G}=f^{-1}(0)$, since $X^{G}$ is a free $\left(S^{1} \times S^{1}\right)$-orbit. Also, $0 \in \mathbb{C}$ is a regular value for $\bar{f}$ : it is easy to find a smooth local section of $\bar{f}$ into the $\left(S^{1} \times S^{1}\right)$-slice $\theta_{1}=\theta_{2}=0$. This again produces a $\left(S^{1} \times S^{1}\right)$-invariant trivialization of the normal bundle to $N=\pi\left(X^{G}\right)=\sqrt{2}\left(e^{i \theta_{1}}, e^{i \theta_{2}}, 0\right)$.

Let $D^{\prime}$ be a small disk around 0 in the image of $f$ and let $D^{\prime \prime}=\hat{\pi}\left(D^{\prime}\right)$. Using homotheties from $D$ to $D^{\prime}$ and $D^{\prime \prime}$ together with the above trivializations permits us to put the map $\pi$ into the form (7.2) locally around $X^{G}$.

Remark 7.11. Statement (2) of Lemma 7.5 does not say that the diffeomorphism type of $X / G$ is functorial. If $h: X \rightarrow X^{\prime}$ is a $G$-equivariant diffeomorphism, the induced homeomorphism $\bar{h}: X / G \rightarrow X^{\prime} / G$ is in general not smooth. For example, take the standard involution $(u, v) \mapsto(-u,-v)$ in $\mathbb{R}^{2}$ and the map $h(u, v)=(u, u+v)$. The induced map $\bar{h}: \mathbb{R}^{2} \rightarrow \mathbb{R}^{2}$ is is determined by the equation $\bar{h} \circ q=q \circ h$, where $q: \mathbb{R}^{2} \rightarrow \mathbb{R}^{2}$ is the complex squaring map $q(u, v)=\left(u^{2}-v^{2}, 2 u v\right)$. Hence, $\bar{h}(x, 0)=(0,2 x)$, if $x \geq 0$, and $\bar{h}(x, 0)=(x, 0)$, if $x<0$. In particular, $\bar{h}$ is not differentiable at $x=0$. The noncompactness of $\mathbb{R}^{2}$ is not the point: one can transport this example onto the Riemann sphere.

Remark 7.12. The smooth structure given by Lemma 7.5 on $X / G$ is not the same as the functional smooth structure on $X / G$ induced by the orbit map (see Bredon [5, p. 301]), nor is it the same as the smooth stratifold structure induced by the $G$-action on $X$ (see Kreck [26]). Both of these structures are functorial, unlike the structure given by Lemma [7.5, but neither one gives $X / G$ the structure of a smooth manifold.

In spite of Remark 7.11, one has the following uniqueness result.

Lemma 7.13. Let $X$ and $X^{\prime}$ be two smooth closed $G$-manifolds with codimension 2 fixed point sets. Suppose that $X$ and $X^{\prime}$ are $G$-equivariantly diffeomorphic. Then, the smooth structures on $X / G$ and $X^{\prime} / G$ given by Lemma 7.5 are diffeomorphic.

Proof. Let $h: X \rightarrow X^{\prime}$ be a $G$-equivariant diffeomorphism and $\bar{h}: X / G \rightarrow X^{\prime} / G$ be the induced homeomorphism. Let $g$ be a $G$-invariant Riemannian metric on $X$ and let $g^{\prime}=h_{*} g$ be the metric on $X^{\prime}$ transported by $h$. With these metrics, $h$ is an isometry and the construction of Step 1 in the proof of Lemma 7.5 implies that $\bar{h}:(X / G)_{g} \rightarrow\left(X^{\prime} / G\right)_{g^{\prime}}$ is a diffeomorphism. The result then follows from Step 2 in the proof of Lemma [7.5,

As an application of the same ideas, we give the following "descent" result for a smooth action of a compact Lie group $H$ on $X$ which commutes with the $G$-action. Note that such an action induces a topological $H$-action on $X / G$.

Lemma 7.14. Let $X$ be a smooth $G$-manifold such that the fixed point set $X^{G}$ is a closed manifold of codimension 2. Suppose that $X$ is equipped with a smooth action of a compact Lie group $H$ which commutes with the $G$-action. Then, there exists a smooth $H$-manifold $M$ and a $H$-equivariant homeomorphism $h: X / G \rightarrow M$ such that the composed map $X \gg X / G \stackrel{h}{\longrightarrow} M$ is a branched covering with branched locus the image of $X^{G}$. If $X$ is closed, the manifold $M$ is unique up to $H$-equivariant diffeomorphism. 
Proof. We use an $H$-invariant Riemannian metric $g$ on $X$ and apply Lemma 7.5 again.

Example 7.15. One of the classical conjugation 4-manifolds is $S^{2} \times S^{2}$ with involution given by complex conjugation on each factor. Note that complex conjugation on $S^{2}$ may be expressed as the reflection $(x, y, z) \mapsto(x, y,-z)$, and this involution commutes with the standard $S^{1}$-action given by rotation in the $x y$-plane. Therefore the quotient $S^{2} \times S^{2} / G$ inherits an effective $T^{2}$-action, which is smooth with respect to the smooth structure provided by Lemma 7.14. However, Orlik [38] applied the classification of smooth $T^{2}$ actions by Orlik and Raymond [39] to show that a smooth homotopy 4-sphere with an effective smooth $T^{2}$-action must be the standard $S^{4}$.

7.16. Lifted structure. We now consider the opposite problem: to show that a smooth structure on the quotient of a 2-fold branched covering induces a canonical smooth $G$ action on the total space.

Lemma 7.17 (Smooth $G$-action). Let $p:(X, Y) \rightarrow(M, N)$ be a branched 2 -fold covering with branched locus $N$, where $M$ is a smooth closed manifold, and $N$ is a smooth closed submanifold of codimension 2 in $M$. Then $X$ admits a smooth $G$-action with $X^{G}=Y$ such that the smooth structure on $X / G$ given by Lemma 7.5 is diffeomorphic to $M$.

Proof. The involution on $X-Y$ is the deck transformation of the covering $X-Y \rightarrow M-N$. This is smooth with respect to the induced smooth structure on $X-Y$ from the covering. Around $Y$, the map $p$ is modelled by (7.2), and we obtain a smooth structure on $X$. The deck transformation of $q: P \times_{O(2)} D^{*} \rightarrow P \overline{\times}_{O(2)} D^{*}$ is given by $(a, z) \mapsto(a,-z)$. It extends to a smooth $G$-action on $X$ with $X^{G}=Y$.

If $M$ is a closed manifold, so is $X$. One has a commutative diagram

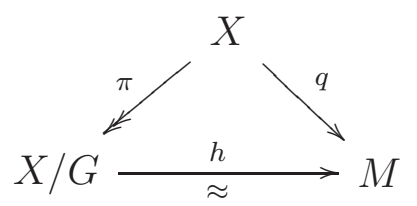

Since $h$ is a continuous bijection between compact spaces, $h$ is a homeomorphism. Hence, $M$ is a smooth structure on $X / G$ satisfying (1) of Lemma 7.5. The result follows from part (2) of the same lemma.

A closed tubular neighbourhood of a codimension 2 submanifold $N \subset M$ will be called a $D$-tube (since it is diffeomorphic to a smooth fibre bundle with fibre $D$ ). The next result is our version of Durfee and Kauffman [9, Prop. 1.1].

Lemma 7.18 (Existence). Let $M$ be a smooth closed manifold, and let $N$ be a smooth closed submanifold of codimension 2 in $M$. Let $\dot{p}: \dot{X} \rightarrow M-N$ be a smooth 2-fold covering. Suppose that, for a D-tube around $N$, the preimage by $\dot{p}$ of each $D^{*}$-fiber is connected. Then

(1) The covering $\dot{p}$ extends to a smooth 2-fold branched covering $p: X \rightarrow M$ with branched locus $N$.

(2) If $p: X \rightarrow M$ and $p^{\prime}: X^{\prime} \rightarrow M$ are two such branched coverings, then $X^{\prime}$ is $G$ diffeomorphic to $X$ (for the smooth structures and $G$-action defined in Lemma 7.17). 
Proof. Choose a Riemannian metric $\bar{g}$ on $M$. This associates a smooth principal $O(2)-$ bundle $Q$ to the normal bundle to $N$. We may suppose that $\bar{g}$ is calibrated around $N$, meaning that the exponential map defines a smooth embedding $\bar{\varphi}: Q \times_{O(2)} D \rightarrow M$. Denote by $\dot{p}: L \rightarrow Q \times_{O(2)} D^{*}$ the 2-fold covering induced from $\dot{p}: \dot{X} \rightarrow M-N$ by the embedding $\bar{\varphi}$. Consider the pull-back diagram

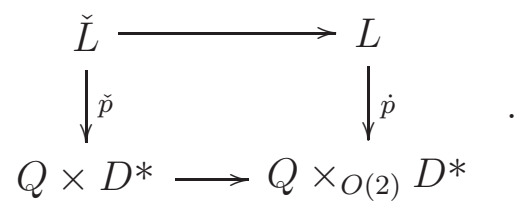

and denote by $\tilde{D}^{*} \rightarrow D^{*}$ the map $z \mapsto z^{2}$ from $D^{*}$ to itself. Choosing a point $a \in Q$ gives a base point $(a, 1) \in Q \times D^{*}$, and we let let $\check{D}^{*}=\check{p}^{-1}\left(\{a\} \times D^{*}\right)$.

The rotation vector field $\xi$ on $D^{*}$, defined by $\xi_{z}=(z, i z) \in D^{*} \times \mathbb{C} \approx T D^{*}$, lifts to a smooth vector field $\check{\xi}$ on $\check{D}^{*}$, which is complete since $\xi$ is. Consider the radius path given by the inclusion $\rho:(0,1] \rightarrow D^{*}$. Choose a lifting $\check{\rho}:(0,1] \rightarrow \check{D}^{*}$ and integrate $\check{\xi}$ with these initial conditions. By our assumption on $D^{*}$-fibers, this will produce a $G$-diffeomorphism $\tilde{D}^{*} \stackrel{\approx}{\longrightarrow} \check{D}^{*}$ over the identity of $D^{*}$.

Over the slice $Q \times\{1\}$, the map $\check{p}$ is a 2 -fold covering $\tilde{Q} \rightarrow Q$. We deduce that there is a $G$-diffeomorphism $\check{\beta}: \tilde{Q} \times_{G} \tilde{D}^{*} \stackrel{\approx}{\longrightarrow}$ over the identity of $Q \times D^{*}$.

Let $a \in Q$ and let $\tilde{a} \in \check{p}^{-1}(a)$. Because of diagram (7.19) and the relation $(a \alpha, z) \sim$ $(a, \alpha z)$ in the definition of $Q \times_{O(2)} D^{*}$, there is a commutative diagram

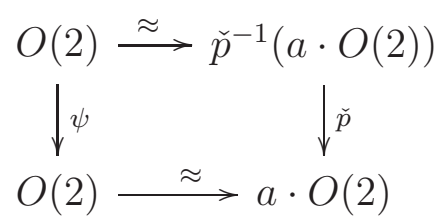

where $\psi$ is the epimorphism defined in (7.1) (this is a 2-fold covering). We deduce that $\tilde{Q}$ is a smooth principal $O(2)$-bundle and that $Q \approx \tilde{Q} \overline{\times}_{O(2)} O(2)$. Hence

$$
Q \times_{O(2)} D^{*} \approx\left[\tilde{Q} \overline{\times}_{O(2)} O(2)\right] \times_{O(2)} D^{*} \approx \widetilde{Q} \overline{\times}_{O(2)} D^{*} .
$$

Suppose first that the normal bundle $\nu$ to $N$ is not orientable. We claim that there is a $G$-diffeomorphism $\beta: \tilde{Q} \times_{O(2)} \tilde{D}^{*} \rightarrow L$ making the following diagram commutative:

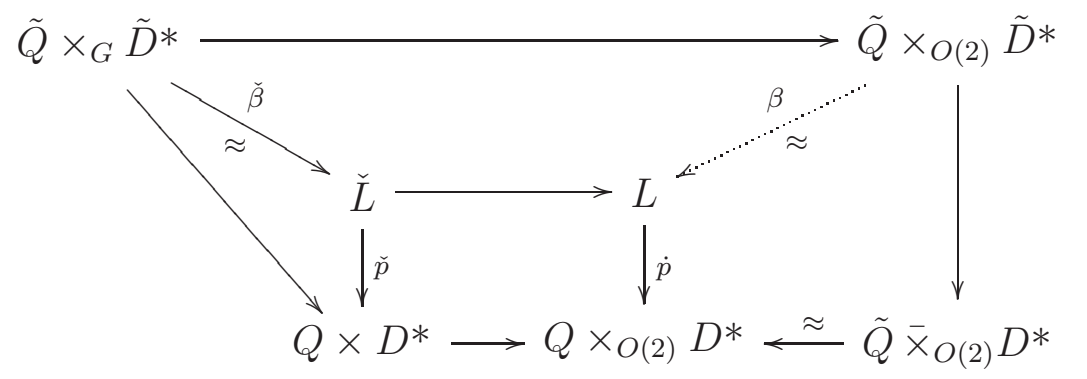


Indeed, if $\nu$ is non-orientable, then $Q$ is connected. As $\tilde{D}$ is connected, we deduce from diagram (7.20) that $\check{L}$ is connected. Hence, any orbit for the diagonal $O(2)$-action on $\tilde{Q} \times_{G} \tilde{D}^{*}$ goes to a single point in $L$. This guarantees that $\check{\beta}$ descends to $\beta$. With these constructions, the covering projection $\dot{p}: \dot{X} \rightarrow M-N$ now extends to a smooth branched covering $p: X \rightarrow M$ where

$$
X=\dot{X} \cup_{\beta} \tilde{Q} \times_{O(2)} D .
$$

This proves the existence of $p: X \rightarrow M$ when $\nu$ is non-orientable. in the other case, we do the whole proof above, replacing $O(2)$ by the connected group $S O(2)$.

For the uniqueness statement of Lemma 7.18, observe that the smooth structure on $X$ given by the decomposition (7.21) is associated to the Riemannian metric $\bar{g}$ on $M$. A proof of the uniqueness statement of Lemma 7.18 may thus be obtained in a process analogous to Steps 2 and 3 of the proof of Lemma 7.5 (see also the uniqueness statement in [9, Prop. 1.1] and its proof).

Remark 7.22. Suppose that, in Lemma 7.18, $N$ is connected and let $V$ be a $D$-tube around $N$. By the homotopy exact sequence of the bundle $D^{*} \rightarrow V-N \rightarrow N$, the condition on the $D$-fibers is equivalent to $\dot{p}^{-1}(V-N)$ being connected. If this is not the case, the proof of Lemma 7.18 shows that $\dot{p}$ extends to an unbranched 2 -fold covering $X \rightarrow M$.

As in Remark 7.11, smooth branched coverings are not functorial, see [9, § 1]. However, as in Lemma 7.13, one has the following uniqueness result.

Lemma 7.23 (Uniqueness). Let $(M, N)$ and $\left(M^{\prime}, N^{\prime}\right)$ be two manifold pairs, where $M$ and $M^{\prime}$ are closed and $N, N^{\prime}$ are closed submanifolds of codimension 2. Suppose that there is a diffeomorphism $h:(M, N) \rightarrow\left(M^{\prime}, N^{\prime}\right)$. Then, the smooth branched coverings over $M$ and and $M^{\prime}$, with branched locus $N$ and $N^{\prime}$, are diffeomorphic.

Proof. Let $X \rightarrow M$ and $X^{\prime} \rightarrow M^{\prime}$ be two such smooth branched coverings. Then the pull-back $h^{*} X^{\prime} \rightarrow M$ is a smooth branched covering over $M$, with branched locus $N$, obviously diffeomorphic to $X^{\prime}$. By Lemma 7.18, $h^{*} X^{\prime}$ is diffeomorphic to $X$.

\section{REFERENCES}

[1] J. F. Adams, On the non-existence of elements of Hopf invariant one, Ann. of Math. (2) 72 (1960), 20-104.

[2] C. Allday and V. Puppe, Cohomological methods in transformation groups, Cambridge Studies in Advanced Mathematics, vol. 32, Cambridge University Press, Cambridge, 1993.

[3] V. I. Arnol'd, The branched covering $\mathbf{C P}^{2} \rightarrow S^{4}$, hyperbolicity and projective topology, Sibirsk. Mat. Zh. 29 (1988), 36-47, 237, translation in Siberian Math. J. 29 (1988), 717-726.

[4] M. F. Atiyah and I. M. Singer, The index of elliptic operators. III, Ann. of Math. (2) 87 (1968), $546-604$.

[5] G. E. Bredon, Introduction to compact transformation groups, Academic Press, New York, 1972, Pure and Applied Mathematics, Vol. 46.

[6] J. Cerf, Sur les difféomorphismes de la sphère de dimension trois $\left(\Gamma_{4}=0\right)$, Lecture Notes in Mathematics, No. 53, Springer-Verlag, Berlin, 1968.

[7] A. Constantin and B. Kolev, The theorem of Kerékjártó on periodic homeomorphisms of the disc and the sphere, Enseign. Math. (2) 40 (1994), 193-204. 
[8] S. K. Donaldson, An application of gauge theory to four-dimensional topology, J. Differential Geom. 18 (1983), 279-315.

[9] A. Durfee and L. Kauffman, Periodicity of branched cyclic covers, Math. Ann. 218 (1975), 157-174.

[10] A. L. Edmonds, Orientability of fixed point sets, Proc. Amer. Math. Soc. 82 (1981), 120-124.

[11] _ _ Aspects of group actions on four-manifolds, Topology Appl. 31 (1989), 109-124.

[12] S. M. Finashin, M. Kreck, and O. Y. Viro, Nondiffeomorphic but homeomorphic knottings of surfaces in the 4-sphere, Topology and geometry-Rohlin Seminar, Lecture Notes in Math., vol. 1346, Springer, Berlin, 1988, pp. 157-198.

[13] S. Finashin, Knotting of algebraic curves in $\mathbb{C P}^{2}$, Topology 41 (2002), 47-55.

[14] R. Fintushel and R. J. Stern, Knots, links, and 4-manifolds, Invent. Math. 134 (1998), 363-400.

[15] R. Fintushel, R. J. Stern, and N. Sunukjian, Exotic group actions on simply connected smooth 4manifolds, (arXiv: 0902.0963 [math.GT]), 2009.

[16] M. Franz and V. Puppe, Steenrod squares on conjugation spaces, C. R. Math. Acad. Sci. Paris 342 (2006), 187-190.

[17] M. H. Freedman, The disk theorem for four-dimensional manifolds, Proceedings of the International Congress of Mathematicians, Vol. 1, 2 (Warsaw, 1983) (Warsaw), PWN, 1984, pp. 647-663.

[18] M. H. Freedman and F. Quinn, Topology of 4-manifolds, Princeton University Press, Princeton, NJ, 1990.

[19] C. H. Giffen, The generalized Smith conjecture, Amer. J. Math. 88 (1966), 187-198.

[20] C. M. Gordon, On the higher-dimensional Smith conjecture, Proc. London Math. Soc. (3) 29 (1974), $98-110$.

[21] _ Knots in the 4-sphere, Comment. Math. Helv. 51 (1976), 585-596.

[22] J.-C. Hausmann and T. Holm, Conjugation spaces and edges of compatible torus actions, (To appear in the proceedings of the conference in honor of H. Duistermaat 70th birthday, arXiv:0807.3289v1), 2008.

[23] J.-C. Hausmann, T. Holm, and V. Puppe, Conjugation spaces, Algebr. Geom. Topol. 5 (2005), 923-964 (electronic).

[24] F. Hirzebruch, The signature of ramified coverings, Global Analysis (Papers in Honor of K. Kodaira), Univ. Tokyo Press, Tokyo, 1969, pp. 253-265.

[25] S. Illman, Smooth equivariant triangulations of $G$-manifolds for $G$ a finite group, Math. Ann. 233 (1978), 199-220.

[26] M. Kreck, Differential algebraic topology, (in preparation: http://www.hausdorff-research-institute.uni-bonn.de/kreck-s

[27] N. H. Kuiper, The quotient space of $\mathbf{C P}(2)$ by complex conjugation is the 4-sphere, Math. Ann. 208 (1974), 175-177.

[28] S. Kwasik, Locally smooth G-manifolds, Amer. J. Math. 108 (1986), 27-37 (1986).

[29] T. Lawson, Splitting $S^{4}$ on $\mathbf{R} P^{2}$ via the branched cover of $\mathbf{C} P^{2}$ over $S^{4}$, Proc. Amer. Math. Soc. 86 (1982), 328-330.

[30] M. Letizia, Quotients by complex conjugation of nonsingular quadrics and cubics in $\mathbf{P}_{\mathbf{C}}^{3}$ defined over R, Pacific J. Math. 110 (1984), 307-314.

[31] D. Lines, Revêtements ramifiés, Enseign. Math. (2) 26 (1980), 173-182.

[32] R. C. Lyndon and P. E. Schupp, Combinatorial group theory, Classics in Mathematics, SpringerVerlag, Berlin, 2001, Reprint of the 1977 edition.

[33] W. S. Massey, The quotient space of the complex projective plane under conjugation is a 4-sphere, Geometriae Dedicata 2 (1973), 371-374.

[34] J. W. Milnor and J. D. Stasheff, Characteristic classes, Princeton University Press, Princeton, N. J., 1974, Annals of Mathematics Studies, No. 76.

[35] M. Olbermann, Conjugations on 6-manifolds, Math. Ann. 342 (2008), 255-271.

[36] _ Conjugations on 6-manifolds with free integral cohomology, arXiv:1001.0911v1 [math.GT], 2010.

[37] _ Involutions on $S^{6}$ with 3-dimensional fixed point set, arXiv:1001.0916v1 [math.GT], 2010.

[38] P. Orlik, Homotopy 4-spheres have little symmetry, Math. Scand. 33 (1973), 275-278. 
[39] P. Orlik and F. Raymond, Actions of the torus on 4-manifolds. I, Trans. Amer. Math. Soc. 152 (1970), 531-559.

[40] P. S. Pao, Nonlinear circle actions on the 4-sphere and twisting spun knots, Topology 17 (1978), 291-296.

[41] V. Puppe, Do manifolds have little symmetry?, J. Fixed Point Theory Appl. 2 (2007), 85-96.

[42] H. Schubert, Knoten mit zwei Brücken, Math. Z. 65 (1956), 133-170.

[43] H. Seifert and W. Threlfall, Seifert and Threlfall: a Textbook of Topology, Pure and Applied Mathematics, vol. 89, Academic Press Inc., New York, 1980.

[44] D. W. Sumners, Smooth $Z_{p}$-actions on spheres which leave knots pointwise fixed, Trans. Amer. Math. Soc. 205 (1975), 193-203.

[45] C. T. C. Wall, Surgery on compact manifolds, second ed., American Mathematical Society, Providence, RI, 1999, Edited and with a foreword by A. A. Ranicki.

[46] G. W. Whitehead, Elements of homotopy theory, Graduate Texts in Mathematics, vol. 61, SpringerVerlag, New York, 1978.

[47] E. C. Zeeman, Twisting spun knots, Trans. Amer. Math. Soc. 115 (1965), 471-495.

Department of Mathematics \& Statistics

MCMASTER University

Hamilton, Ontario L8S 4K1, Canada

E-mail address: hambleton@mcmaster.ca

Section de Mathématiques

Université De Genève, B.P. 240

CH-1211 Genève 24, Switzerland

E-mail address: Jean-Claude.Hausmann@unige.ch 